\title{
Stellar pulsations excited by a scattered mass
}

\author{
V. Ferrari ${ }^{1}$, L. Gualtieri ${ }^{2}$, A. Borrelli ${ }^{3}$ \\ 1 Dipartimento di Fisica "G.Marconi", Università di Roma "La Sapienza" \\ and \\ Sezione INFN ROMA1, p.le A. Moro 5, I-00185 Roma, Italy \\ ${ }^{2}$ Dip. di Fisica Teorica, Università di Torino, 10125, Italy \\ 3 Dip. di Fisica, Università di Pavia, Pavia, 27100, Italy
}

(October 21, 2018)

\begin{abstract}
We compute the energy spectra of the gravitational signals emitted when a mass $m_{0}$ is scattered by the gravitational field of a star of mass $M>>m_{0}$. We show that, unlike black holes in similar processes, the quasi-normal modes of the star are excited, and that the amount of energy emitted in these modes depends on how close the exciting mass can get to the star.
\end{abstract}

PACS numbers: 04.30.-w, 04.40.Dg

Typeset using REVTEX 


\section{INTRODUCTION}

In this paper we study the gravitational signals emitted when a mass $m_{0}$ is scattered by the gravitational field of a star of mass $M$. Under the assumption that $m_{0}$ is much smaller than $M$, this study can be done in the framework of a first order perturbation theory, by assuming that the perturbations of the gravitational field and of the fluid composing the star are excited by the stress-energy tensor of a point-like scattered mass (a particle).

Since 1971, when M.Davis, R.Ruffini, W.H.Press and R.H.Price [1] computed the energy and the waveform emitted when a particle is radially captured by a Schwarzschild black hole, the perturbations of rotating and nonrotating black holes excited by infalling, scattered, or orbiting masses, have been extensively investigated. No comparable attention has been dedicated to the study of stellar perturbations excited by small masses, although this problem is certainly interesting. Indeed, we know that neutron stars exist, and observations allow to infer the location and the mass of many of them, as well as their rotation periods. In addition, the worldwide experimental effort spent to detect gravitational waves, will hopefully be crowned by success in a not too far future. Therefore, it is time to gain new theoretical insight on the characteristics of the gravitational signals emitted by neutron stars. Until now, the application of the theory of stellar perturbations has mainly regarded the determination of the frequencies of the quasi-normal modes, at which stars are expected to pulsate and emit gravitational waves. However, whether these modes can be excited, and how much energy is emitted at the corresponding frequencies, can be understood only by considering realistic astrophysical situations. The theory of stellar perturbations, suitably matched with the theory of black hole perturbations, allows to explore this new field.

In our investigation we shall consider a polytropic star with mass and radius typical of neutron stars, and integrate the equations describing the scattering of a small mass. In the interior of the star, we shall use the equations for the axial and polar perturbations derived by S.Chandrasekhar \& V.Ferrari in 1990 in ref. [2], (to be referred to hereafter as Paper I), that 
hold also in a more general gauge appropriate to describe non-axisymmetric perturbations. Outside the star, one may continue the solution by integrating the Regge-Wheeler and the Zerilli equations [3], [4], with a source term given by the stress energy tensor of the scattered mass moving along a geodesic of the unperturbed spacetime. However, it is known that the source term of these equations diverges when the mass reaches the periastron, and therefore we switch to a different formalism, and use the generalized non-homogeneous Regge-Wheeler equation, which was introduced by T.Nakamura and M.Sasaki [5] to overcome this problem. We shall integrate this equation by adopting the procedure discussed in ref. [6] (to be referred to hereafter as Paper II), and further developed in this paper. We shall show how the problem of matching the interior and exterior solution can be solved, and find the energy spectra of the emitted radiation for different values of the orbital parameters of the scattered mass.

The plan of the paper is the following. In section II we shall briefly review the equations to be integrated in the interior of the star; in section III the equations governing the exterior perturbations will be shown; the procedure to find the complete solution and the matching conditions will be discussed in section IV; in section $\mathrm{V}$ we will describe a model of polytropic star for which we shall compute the characteristic oscillation frequencies; in section VI we will show the energy spectra emitted when a small mass is scattered by such star.

\section{THE EQUATIONS DESCRIBING THE PERTURBED SPACETIME INSIDE THE STAR.}

In order to describe the perturbations induced by a mass scattered by a spherical, nonrotating star, we choose a gauge appropriate to describe non-axisymmetric perturbations

$$
\begin{aligned}
d s^{2} & =e^{2 \nu(r)} d t^{2}-e^{2 \mu_{2}(r)} d r^{2}-r^{2} d \vartheta^{2}-r^{2} \sin ^{2} \vartheta d \varphi^{2} \\
& +\sum_{\ell m} \int_{-\infty}^{+\infty} d \omega e^{-i \omega t}\left\{2 e^{2 \nu} N_{\ell m}(r, \omega) Y_{\ell m}(\vartheta, \varphi) d t^{2}-2 e^{2 \mu_{2}} L_{\ell m}(r, \omega) Y_{\ell m}(\vartheta, \varphi) d r^{2}\right. \\
& -2 r^{2} H_{33} d \vartheta^{2}-2 r^{2} \sin ^{2} \vartheta H_{11} d \varphi^{2}-2 r^{2} H_{13} d \vartheta d \varphi+h_{\ell m}^{0}(r, \omega) \sin \theta Y_{\ell m}(\vartheta, \varphi) d t d \varphi \\
& +h_{\ell m}^{1}(r, \omega) \sin \theta \frac{\partial Y_{\ell m}(\vartheta, \varphi)}{\partial \vartheta} d r d \varphi-h_{\ell m}^{0}(r, \omega) \frac{1}{\sin \theta} \frac{\partial Y_{\ell m}(\vartheta, \varphi)}{\partial \varphi} d t d \vartheta
\end{aligned}
$$




$$
\left.-h_{\ell m}^{1}(r, \omega) \frac{1}{\sin \theta} \frac{\partial Y_{\ell m}(\vartheta, \varphi)}{\partial \varphi} d r d \vartheta\right\}
$$

where

$$
\begin{aligned}
& H_{11}=\left[T_{\ell m}(r, \omega)+V_{\ell m}(r, \omega)\left(\frac{1}{\sin ^{2} \vartheta} \frac{\partial^{2}}{\partial \varphi^{2}}+\cot \vartheta \frac{\partial}{\partial \vartheta}\right)\right] Y_{\ell m}(\varphi, \vartheta) \\
& H_{13}=V_{\ell m}(r, \omega)\left[\frac{\partial^{2}}{\partial \varphi \partial \vartheta}-\frac{\partial}{\partial \varphi} \cot \vartheta\right] Y_{\ell m} \\
& H_{33}=\left[T_{\ell m}(r, \omega)+V_{\ell m}(r, \omega) \frac{\partial^{2}}{\partial \vartheta^{2}}\right] Y_{\ell m}(\varphi, \vartheta) .
\end{aligned}
$$

and $Y_{\ell m}(\vartheta, \varphi)$ are the scalar spherical harmonics. The perturbed part of the metric (2.1) has been Fourier-expanded, and the usual decomposition in tensor spherical harmonics has been performed (cfr. [3, [4]). The functions $\left[N_{\ell m}(r, \omega), L_{\ell m}(r, \omega), V_{\ell m}(r, \omega), T_{\ell m}(r, \omega)\right]$, are the radial part of the polar, (even) metric components, whereas $h_{\ell m}^{0}(r, \omega)$ and $h_{\ell m}^{1}(r, \omega)$ are the axial (odd) part. It should be mentioned that in the axisymmetric case $(m=0)$ the previous gauge reduces to that used in Paper I for the polar perturbations, and to the Regge-Wheeler gauge [3] for the axial ones.

The unperturbed metric functions $\nu(r)$ and $\mu_{2}(r)$ have to be determined by numerically integrating the Einstein equations coupled to the equations of hydrostatic equilibrium, for an assigned equation of state. Under the assumption that the star is composed by a perfect fluid with energy-momentum tensor given by $T^{\alpha \beta}=(p+\epsilon) u^{\alpha} u^{\beta}-p g^{\alpha \beta}$, where $p$ and $\epsilon$ are, respectively, the pressure and the energy density, the relevant equations are

$$
\begin{aligned}
& {\left[1-\frac{2 m(r)}{r}\right] p_{, r}=-(\varepsilon+p)\left[p r+\frac{m(r)}{r^{2}}\right]} \\
& \nu=-\int_{0}^{r} \frac{p_{, r}}{(\epsilon+p)} d r+\nu_{0}, \quad e^{2 \mu_{2}}=\left(1-\frac{2 m(r)}{r}\right)^{-1},
\end{aligned}
$$

where $m(r)=\int_{0}^{r} \varepsilon r^{2} d r$ is the mass contained within a sphere of radius $r$. The solution for the function $\nu(r)$ requires the determination of the constant $\nu_{0}$, which can be found by imposing that at the boundary of the star the metric reduces to the Schwarzschild metric

$$
\left(e^{2 \nu}\right)_{r=R}=\left(e^{-2 \mu_{2}}\right)_{r=R}=1-2 M / R,
$$


where $M=m(R)$ is the total mass.

It is easy to check that, since $Y_{\ell m}(\vartheta, \varphi)=(-1)^{m} Y_{\ell-m}^{*}(\vartheta, \varphi)$, the perturbed metric functions satisfy the following property

$$
F_{\ell m}(r, \omega)=(-1)^{m} F_{\ell-m}^{*}(r,-\omega),
$$

where $F_{\ell m}(r, \omega)$ is any of the functions $\quad\left[N_{\ell m}(r, \omega), L_{\ell m}(r, \omega), V_{\ell m}(r, \omega), T_{\ell m}(r, \omega)\right]$, and $\left[h_{\ell m}^{0}(r, \omega), h_{\ell m}^{1}(r, \omega)\right]$.

By writing explicitely the perturbed Einstein's equations coupled to the hydrodynamical equations in the interior of the star, it is possible to verify that the resulting separated equations coincide with those given in Paper I, both for the polar and for the axial perturbations. As a consequence, the decoupling of the gravitational perturbations from the perturbations of the thermodynamical variables that was performed in Paper I for the polar equations, is possible also in the present case of non-axisymmetric perturbations. Therefore, the equations to integrate inside the star, to find the values of the polar and axial functions at the boundary, are the following. If we consider stars with a barotropic equation of state, the polar equations are (cfr. eqs. (72)-(75) of Paper I)

$$
\begin{aligned}
& X_{, r, r}+\left(\frac{2}{r}+\nu_{, r}-\mu_{2, r}\right) X_{, r}+\frac{n}{r^{2}} e^{2 \mu_{2}}(N+L)+\omega^{2} e^{2\left(\mu_{2}-\nu\right)} X=0, \\
& \left(r^{2} G\right), r=n \nu_{, r}(N-L)+\frac{n}{r}\left(e^{2 \mu_{2}}-1\right)(N+L)+r\left(\nu_{, r}-\mu_{2, r}\right) X_{, r}+\omega^{2} e^{2\left(\mu_{2}-\nu\right)} r X, \\
& -\nu_{, r} N_{, r}=-G+\nu_{, r}\left[X_{, r}+\nu_{, r}(N-L)\right]++\frac{1}{r^{2}}\left(e^{2 \mu_{2}}-1\right)\left(N-r X_{, r}-r^{2} G\right) \\
& -e^{2 \mu_{2}}(\epsilon+p) N+\frac{1}{2} \omega^{2} e^{2\left(\mu_{2}-\nu\right)}\left\{N+L+\frac{r^{2}}{n} G+\frac{1}{n}\left[r X_{, r}+(2 n+1) X\right]\right\}, \\
& -L_{, r}=(N+2 X)_{, r}+\left(\frac{1}{r}-\nu_{, r}\right)(-N+3 L+2 X)+ \\
& +\left[\frac{2}{r}-(Q+1) \nu_{, r}\right]\left[N-L+\frac{r^{2}}{n} G+\frac{1}{n}\left(r X_{, r}+X\right)\right],
\end{aligned}
$$

where $Q=\epsilon_{, r} / p_{, r}$, the function $V$ has been replaced by $X=n V$, with $n=(\ell-1)(\ell+2) / 2$, and the function $G$ takes the place of $T$

$$
\begin{aligned}
G=\nu_{, r}\left[\frac{n+1}{n} X-T\right]_{, r} & +\frac{1}{r^{2}}\left(e^{2 \mu_{2}}-1\right)[n(N+T)+N]+\frac{\nu_{, r}}{r}(N+L)- \\
& -e^{2 \mu_{2}}(\epsilon+p) N+\frac{1}{2} \omega^{2} e^{2\left(\mu_{2}-\nu\right)}\left[L-T+\frac{2 n+1}{n} X\right] .
\end{aligned}
$$


Outside the star, where $\epsilon$ and $p$ vanish, these equations become appropriate to describe the Schwarzschild perturbations, and can be reduced to the Zerilli equation [4]

$$
\frac{d^{2} Z^{p o l}}{d r_{*}^{2}}+\left\{\omega^{2}-\frac{2(r-2 M)}{r^{4}(n r+3 M)^{2}}\left[n^{2}(n+1) r^{3}+3 M n^{2} r^{2}+9 M^{2} n r+9 M^{3}\right]\right\} Z^{p o l}=0 .
$$

The value of the Zerilli function at the surface, $Z^{\text {pol }}(\omega, R)$, can be found in terms of the solution of the eqs. (2.6) as follows (cfr. eq. (93) Paper I)

$$
Z^{p o l}(\omega, R)=\frac{R}{n R+3 M}\left(\frac{3 M}{n} X(\omega, R)-R L(\omega, R)\right)
$$

and similarly for its first derivative. For simplicity, in eqs. (2.6-2.9) we have omitted the harmonic indices $(\ell m)$.

The equations for the axial perturbations can be reduced to a single wave equation (cfr. eqs. (148)and (149), Paper I)

$$
\frac{d^{2} Z_{\ell m}^{a x}}{d r_{*}^{2}}+\left\{\omega^{2}-\frac{e^{2 \nu}}{r^{3}}\left[\ell(\ell+1) r+r^{3}(\epsilon-p)-6 m(r)\right]\right\} Z_{\ell m}^{a x}=0
$$

where $r_{*}=\int_{0}^{r} e^{-\nu+\mu_{2}} d r$, and the function $Z_{\ell m}^{a x}$ is related to the axial metric components by

$$
h_{\ell m}^{0}=\frac{2 i}{\omega} \frac{d}{d r_{*}}\left(r Z_{\ell m}^{a x}\right), \quad \quad h_{\ell m}^{1}=2 e^{-2 \nu}\left(r Z_{\ell m}^{a x}\right)
$$

Outside the star eq. (2.10) reduces to the Regge-Wheeler equation.

\section{THE EQUATIONS DESCRIBING THE PERTURBED SPACETIME OUTSIDE THE STAR.}

The metric exterior to a nonrotating star reduces to the Schwarzschild metric, and therefore the perturbed spacetime is described by the perturbations of a Schwarzschild black hole. When the source exciting the perturbations is a scattered mass, the source term of the Regge-Wheeler and of the Zerilli equations diverge at the periastron (where $\frac{d r}{d \tau}=0$ ), and alternative equations have to be used. In 1973 J.M. Bardeen and W.H. Press derived a wave equation with a complex potential barrier for the Weyl scalars $\delta \Psi_{0}$ and $\quad r^{4} \delta \Psi_{4}$ 
[7]. This equation was subsequently generalized by S.A. Teukolsky [8], who found a master equation governing the electromagnetic, gravitational and neutrino-field perturbations of a Kerr black hole, which reduces to the Bardeen-Press equation when the rotation parameter is set to zero. We shall refer to the Bardeen-Press-Teukolsky equation as the BPT equation. After separating the variables, the radial BPT equation can be written as

$$
\left\{\Delta^{2} \frac{d}{d r}\left[\frac{1}{\Delta} \frac{d}{d r}\right]+\left[\frac{\left(r^{4} \omega^{2}+4 i(r-M) r^{2} \omega\right)}{\Delta}-8 i \omega r-2 n\right]\right\} \Psi_{\ell m}^{B P T}(\omega, r)=-T_{\ell m}(\omega, r),
$$

where $\Delta=r^{2}-2 M r$, the BPT function is related to $\delta \Psi_{4}$ by

$$
\delta \Psi_{4}(t, r, \vartheta, \varphi)=\frac{1}{r^{4}} \sum_{\ell m} \int_{-\infty}^{+\infty} d \omega e^{-i \omega t}{ }_{-2} S_{\ell m}(\vartheta, \varphi) \Psi_{\ell m}^{B P T}(\omega, r),
$$

and ${ }_{-2} S_{\ell m}(\vartheta, \varphi)$, in terms of which the separation is accomplished, is the spin-weighted spherical harmonic

$$
\begin{array}{r}
{ }_{-2} S_{\ell m}(\vartheta, \varphi)=\frac{1}{2 \sqrt{n(n+1)}}\left[\frac{\partial^{2} Y_{\ell m}(\vartheta, \varphi)}{\partial \vartheta^{2}}-\cot \vartheta \frac{\partial Y_{\ell m}(\vartheta, \varphi)}{\partial \vartheta}+\frac{m^{2}}{\sin ^{2} \vartheta} Y_{\ell m}(\vartheta, \varphi)\right. \\
\left.+\frac{2 m}{\sin \vartheta}\left(\frac{\partial Y_{\ell m}(\vartheta, \varphi)}{\partial \vartheta}-Y_{\ell m}(\vartheta, \varphi) \cot \vartheta\right)\right] .
\end{array}
$$

The source $T_{\ell m}(\omega, r)$ is given by

$$
T_{\ell m}(\omega, r)=-2 r^{4} \sqrt{n(n+1)} \tilde{T}_{\ell m}^{n n}(\omega, r)+2 \sqrt{n} \Delta \Lambda_{+} \frac{r^{5}}{\Delta} \tilde{T}_{\ell m}^{n \bar{m}}(\omega, r)-\frac{\Delta}{2 r} \Lambda_{+} \frac{r^{6}}{\Delta} \Lambda_{+} r \tilde{T}_{\ell m}^{n n}(\omega, r),
$$

where

$$
\Lambda_{+}=\frac{d}{d r_{*}}+i \omega
$$

and the factors $\tilde{T}_{a b}^{\ell m \omega}(r)$ are the radial parts of Newman-Penrose components of the stressenergy tensor (see Paper II, app. A.2). In the present paper we prefer to use another equation, related to the BPT equation, which was derived by Nakamura and Sasaki [5]. They showed that, given a solution of the inhomogeneous BPT equation, there exists a function $Z_{\ell m}^{N S}(\omega, r)$ related to $\Psi_{\ell m}^{B P T}(\omega, r)$ by the Chandrasekhar transformation [9] 


$$
\Psi_{\ell m}^{B P T}(\omega, r)=D_{C H} Z_{\ell m}^{N S}(\omega, r)
$$

where

$$
D_{C H}=\Delta \Lambda_{+} \frac{r^{2}}{\Delta} \Lambda_{+} r
$$

which satisfies the generalized inhomogeneous Regge-Wheeler equation

$$
\left\{\frac{d^{2}}{d r_{*}^{2}}-\left[\omega^{2}-\frac{\Delta}{r^{5}}[\ell(\ell+1)-6 M]\right]\right\} Z_{\ell m}^{N S}=S_{\ell m}^{N S} .
$$

The source term is related to the source of the BPT equation by

$$
D_{C H}\left(\frac{r^{4}}{\Delta} S_{\ell m}^{N S}\right)=-T_{\ell m}
$$

We shall now write the source term of the Nakamura-Sasaki equation explicitely. If we use, as in paper I, the convenction $G_{\mu \nu}=2 T_{\mu \nu}$, the stress-energy tensor of a point-like mass $m_{0}$, moving along a geodesic $\underline{r}(\tau)$ of the unperturbed spacetime, is

$$
T^{\mu \nu}=2 m_{0} \int d \tau \frac{d z^{\mu}}{d \tau} \frac{d z^{\nu}}{d \tau} \delta^{(4)}(\underline{r}-\underline{r}(\tau))
$$

Since

$$
\delta(\bar{r}-r(\tau))=\sum_{i} \frac{1}{\left|\frac{d r}{d \tau}\right|_{\tau_{i}(\bar{r})}} \delta\left(\tau-\tau_{i}(\bar{r})\right),
$$

where $\tau_{i}(r)$ are the solution of $r(\tau)=\bar{r}$, the solution can be divided in two branches, corresponding to the incoming and outgoing part of the trajectory. Consequently, the stressenergy tensor can be written as

$$
T^{\mu \nu}=2 m_{0} \sum_{i=1}^{1} \frac{2}{r^{2}|\gamma|} \frac{d x^{\mu}}{d \tau} \frac{d x^{\nu}}{d \tau} \delta\left(t-t_{i}(r)\right) \delta^{(2)}\left(\Omega-\Omega_{i}(r)\right),
$$

where $t_{i}(r), \Omega_{i}(r)$ are the time and angular position of the particle on the $\mathrm{i}-$ eth branch of the trajectory. In terms of the tensor (3.11), the source of the BPT equation can be written as

$$
\begin{gathered}
T_{\ell m}(\omega, r)=-m_{0}\left\{\frac{\Delta^{2}}{r^{2}} \sqrt{n(n+1)} V^{\prime 2}(r){ }_{0} f_{\ell m}(r) e^{i \omega V(r)}+\right. \\
\left.+\sqrt{2 n} \Delta \frac{\partial}{\partial r_{*}}\left(r^{2} V^{\prime}(r){ }_{-1} f_{\ell m}(r) e^{i \omega V(r)}\right)+\frac{\Delta}{2 r} \frac{\partial}{\partial r_{*}}\left[\frac{r^{6}}{\Delta} \frac{\partial}{\partial r_{*}}\left(r_{-2} f_{\ell m}(r) e^{i \omega V(r)}\right)\right]\right\} e^{-i \omega r_{*}} .
\end{gathered}
$$


where

$$
\begin{aligned}
& V(r)=t(r)+r_{*}, \\
& { }_{0} f_{l m}(r)=|\gamma|{ }_{0} S_{\ell m}^{*}(\Omega(r)), \\
& { }_{-1} f_{l m}(r)=\sigma\left(\frac{d \vartheta}{d \tau}-i \sin \vartheta \frac{d \varphi}{d \tau}\right)_{\Omega(r)}{ }_{-1} S_{\ell m}^{*}(\Omega(r)), \\
& { }_{-2} f_{l m}(r)=\frac{1}{|\gamma|}\left(\frac{d \vartheta}{d \tau}-i \sin \vartheta \frac{d \varphi}{d \tau}\right)_{\Omega(r)}^{2}-{ }_{2} S_{\ell m}^{*}(\Omega(r)),
\end{aligned}
$$

the prime indicates differentiation with respect to $\mathrm{r}$, and ${ }_{-0} S_{\ell m}^{*}(\Omega(r)),{ }_{-1} S_{\ell m}^{*}(\Omega(r))$ are the complex conjugate of the spin-weighted sperical harmonics of weight 0 and 1 , and $\sigma=$ $\operatorname{sign}\left[\frac{d r}{d \tau}\right]$. We shall assume that a particle $m_{0}$ starts its journey at radial infinity with energy $E$ and angular momentum $L_{z}$, and we shall put $\varphi\left(r_{t}\right)=\tau\left(r_{t}\right)=0$, at the turning point $r=r_{t}$. We choose $E$ and $L_{z}$, such that the particle is scattered by the star, and follows a geodesic on the plane $\vartheta=\frac{\pi}{2}$, described by the equations

$$
\frac{d t}{d \tau}=\frac{E}{1-\frac{2 M}{r}}, \quad \frac{d r}{d \tau} \equiv \gamma= \pm \sqrt{E^{2}-\left(1-\frac{2 M}{r}\right)\left(1+\frac{L_{z}^{2}}{r^{2}}\right)}, \quad \frac{d \varphi}{d \tau}=\frac{L_{z}}{r^{2}}
$$

The source term of the Nakamura-Sasaki equation can be derived from eqs. (3.9), (3.12) and (3.13), as in 15. The result is

$$
\begin{aligned}
& S_{\ell m}^{N S}(\omega, r)=\frac{\Delta}{r^{5}} e^{-i \omega r_{*}}\left\{\left[A_{\ell m}^{1}(\omega, r)+i B_{\ell m}^{1}(\omega, r)\right]\right. \\
& \left.+\int_{r}^{\infty} d r^{\prime}\left[A_{\ell m}^{2}(\omega, r)+i B_{\ell m}^{2}(\omega, r)\right]+\int_{r}^{\infty} d r^{\prime} \int_{r^{\prime}}^{\infty} d r^{\prime \prime}\left[A_{\ell m}^{3}(\omega, r)+i B_{\ell m}^{3}(\omega, r)\right]\right\}
\end{aligned}
$$

where

$$
\begin{aligned}
& A_{\ell m}^{j}=\left[a_{\ell m}^{j} \cos \omega r_{*}-b_{\ell m}^{j} \sin \omega r_{*}\right], \\
& B_{\ell m}^{j}=\left[b_{\ell m}^{j} \cos \omega r_{*}+a_{\ell m}^{j} \sin \omega r_{*}\right],
\end{aligned}
$$

with $\mathrm{j}=1,2,3$, 


$$
\left\{\begin{array}{l}
a_{\ell m}^{1}=\left[K_{\ell m}^{0} \gamma-\frac{K_{\ell m}^{2}}{\gamma}\right] \cos (\omega t-m \varphi), \\
b_{\ell m}^{1}=-\bar{C}_{\ell m}^{1} \sin (\omega t-m \varphi) \\
a_{\ell m}^{2}=\left[K_{\ell m}^{0} \gamma^{\prime}-\frac{2}{\gamma r} K_{\ell m}^{2}\right] \cos (\omega t-m \varphi)+K_{\ell m}^{0} \frac{m L_{z}}{r^{2}} \sin (\omega t-m \varphi) \\
b_{\ell m}^{2}=\frac{m L_{z}}{\gamma r^{2}} \bar{C}_{\ell m}^{1} \cos (\omega t-m \varphi) \\
a_{\ell m}^{3}=\left[K_{\ell m}^{0} \frac{\omega m L_{z}}{\gamma \Delta}-\frac{2 K_{\ell m}^{2}}{\gamma r^{2}}\right] \cos (\omega t-m \varphi)+\frac{2 K_{\ell m}^{0} \omega M r^{2}}{\Delta^{2}} \sin (\omega t-m \varphi) \\
b_{\ell m}^{3}=K_{\ell m}^{0} \omega\left[-\frac{2 M r^{2} \gamma}{\Delta^{2}}+\frac{\gamma^{\prime} r^{2}}{\Delta}\right] \cos (\omega t-m \varphi)+\frac{K_{\ell m}^{0} \omega m L_{z}}{\Delta} \sin (\omega t-m \varphi)
\end{array}\right.
$$

The constants $K_{0}, \ldots$ are

$$
\left\{\begin{array}{l}
K_{\ell m}^{0}=-\mu \frac{\sqrt{\lambda(\lambda+2)}}{\omega^{2}}{ }_{0} S_{\ell m}^{*}\left(\frac{\pi}{2}, 0\right) \\
K_{\ell m}^{1}=\mu \frac{2 i L_{z} \sqrt{\lambda}}{\omega}{ }_{-1} S_{\ell m}^{*}\left(\frac{\pi}{2}, 0\right), \quad \bar{C}_{\ell m}^{1}=-i K_{\ell m}^{1} \\
K_{\ell m}^{2}=\mu L_{z-2}^{2} S_{\ell m}^{*}\left(\frac{\pi}{2}, 0\right),
\end{array}\right.
$$

and

$$
\lambda=\ell(\ell+1) .
$$

\section{THE SOLUTION OF THE NAKAMURA-SASAKI EQUATION AND THE MATCHING CONDITIONS.}

In order to integrate the Nakamura-Sasaki equation (3.8) outside the star, we need to know the value of $Z_{\ell m}^{N S}$ and its derivative at the surface $r=R$. The Nakamura-Sasaki function is related to the solution of the Regge-Wheeler equation for the axial perturbations, $Z^{a x}$, and to that of the Zerilli equation for the polar ones, $Z^{\text {pol }}$, by the following relations 6

$$
Z_{\ell m}^{N S}(\omega, r)=Z_{\ell m}^{1}(\omega, r)+i Z_{\ell m}^{2}(\omega, r)
$$

where

$$
\begin{aligned}
& Z_{\ell m}^{1}(\omega, r)=-\frac{\sqrt{n(n+1)}}{4(k-2 i \omega \beta)}\left[\left(k+2 \beta^{2} f\right) Z_{\ell m}^{p o l}(\omega, r)-2 \beta Z_{\ell m, r_{*}}^{p o l}(\omega, r)\right], \\
& Z_{\ell m}^{2}(\omega, r) \equiv \frac{\sqrt{n(n+1)}}{4 i \omega} Z_{\ell m}^{a x}(\omega, r)
\end{aligned}
$$


and

$$
\left\{\begin{array}{l}
\beta=6 M, \\
k=4 n(n+1) \\
f=\frac{\Delta}{r^{3}[(\ell-1)(\ell+2) r+6 M]} .
\end{array}\right.
$$

It should be stressed that both $Z_{\ell m}^{1}(\omega, r)$ and $Z_{\ell m}^{2}(\omega, r)$ satisfy the Regge-Wheeler equation. Thus, $Z_{\ell m}^{N S}(\omega, R)$ and its derivative can be found in terms of $Z^{p o l}(\omega, R)$ and $Z^{a x}(\omega, R)$ and their first derivatives. These values can be found by numerically integrating the equations for the polar and axial perturbations in the interior of the star (cfr. eqs. 2.9 and 2.10), by imposing the boundary conditions that, for each assigned value of the frequency, the solution is regular near the origin, and that the perturbation of the thermodynamical variables vanish at the boundary, so that the perturbed spacetime reduces to vacuum (cfr. Paper I, sec. 6,7 and 11). However, these conditions do not define the amplitude of the functions $Z^{\text {pol }}(\omega, R)$ and $Z^{a x}(\omega, R)$, which depends on the exciting source. If we name $\bar{Z}_{\ell m}^{p o l}(\omega, R)$ and $\bar{Z}_{\ell m}^{a x}(\omega, R)$ the values found by integrating the interior equations with an arbitrary amplitude at the centre of the star, the 'true' values of $Z^{\text {pol }}(\omega, R)$ and $Z^{a x}(\omega, R)$ will be

$$
\left\{\begin{array}{l}
Z_{\ell m}^{p o l}(\omega, R)=\chi_{\ell m}^{1}(\omega) \bar{Z}_{\ell m}^{p o l}(\omega, R) \\
Z_{\ell m}^{a x}(\omega, R)=\chi_{\ell m}^{2}(\omega) \bar{Z}_{\ell m}^{a x}(\omega, R)
\end{array},\right.
$$

and $\chi_{\ell m}^{1}(\omega)$ and $\chi_{\ell m}^{2}(\omega)$ have to be found. Consequently, the functions $Z_{\ell m}^{1}(\omega, R)$ and $Z_{\ell m}^{2}(\omega, R)$ defined in eqs. (4.2) suffer the same ambiguity

$$
\left\{\begin{array}{l}
Z_{\ell m}^{1}(\omega, R)=\chi_{\ell m}^{1}(\omega) \bar{Z}_{\ell m}^{1}(\omega, R) \\
Z_{\ell m}^{2}(\omega, R)=\chi_{\ell m}^{2}(\omega) \bar{Z}_{\ell m}^{2}(\omega, R)
\end{array},\right.
$$

and $\bar{Z}_{\ell m}^{1}(\omega, R), \bar{Z}_{\ell m}^{2}(\omega, R)$ are (cfr. eqs. 4.2)

$$
\begin{aligned}
& \bar{Z}_{\ell m}^{1}(\omega, R)=-\frac{\sqrt{n(n+1)}}{4(k-2 i \omega \beta)}\left[\left(k+2 \beta^{2} f\right) \bar{Z}_{\ell m}^{p o l}(\omega, R)-2 \beta \bar{Z}_{\ell m, r_{*}}^{p o l}(\omega, R)\right], \\
& \bar{Z}_{\ell m}^{2}(\omega, R)=\frac{\sqrt{n(n+1)}}{4 i \omega} \bar{Z}_{\ell m}^{a x}(\omega, R),
\end{aligned}
$$

$\chi_{\ell m}^{1}(\omega)$ and $\chi_{\ell m}^{2}(\omega)$ determine how much of the polar and of the axial modes are excited by the particular source we are considering. 
Eq. (3.8) has to be solved by imposing that, at the surface, the solution of the perturbed equations in the interior matches continuously with the exterior solution, which, in addition, has to behave as a pure outgoing wave at radial infinity, i.e.

$$
\left\{\begin{array}{l}
L_{R W} Z_{\ell m}^{N S}(\omega, r)=S_{\ell m}^{N S}(\omega, r) \\
Z_{\ell m}^{N S}(\omega, R)=Z_{\ell m}^{1}(\omega, R)+i Z_{\ell m}^{2}(\omega, R) \\
Z^{N S^{\prime}{ }_{l m}}(\omega, R)=Z_{\ell m}^{1^{\prime}}(\omega, R)+i Z_{\ell m}^{2^{\prime}}(\omega, R) \\
Z_{\ell m}^{N S} \sim e^{i \omega r_{*}} \quad r_{*} \rightarrow \infty
\end{array}\right.
$$

where the prime indicates differentiation with respect to $r_{*}$, and $L_{R W}$ is the ReggeWheeler operator

$$
L_{R W} \equiv \frac{d^{2}}{d r_{*}^{2}}-\left\{\omega^{2}-\frac{\Delta}{r^{5}}[\ell(\ell+1)-6 M]\right\} .
$$

The solution of eqs. (4.8) can be found by defining the Green's function appropriate to the problem, as explained in Paper II. Here we briefly summarize the procedure. For $r \geq R$ we define the function $Y_{\ell}\left(\omega, r_{*}\right)$, which satisfies the homogeneous Regge-Wheeler equation with the pure outgoing wave condition at infinity

$$
\left\{\begin{array}{l}
L_{R W} Y_{\ell}\left(\omega, r_{*}\right)=0 \\
Y_{\ell}(\omega, \infty)=e^{i \omega r_{*}}
\end{array}\right.
$$

and the functions $\bar{Z}_{\ell}^{1}\left(\omega, r_{*}\right)$ and $\bar{Z}_{\ell}^{2}\left(\omega, r_{*}\right)$ that, as $Y_{\ell}\left(\omega, r_{*}\right)$, satisfy a homogeneous Regge-Wheeler equation, with boundary condition at the surface of the star given by eqs. (4.6) and (4.7), respectively. It should be noted that, since the Regge-Wheeler operator does not depend on $m$, the functions $Y_{\ell}\left(\omega, r_{*}\right), \bar{Z}_{\ell}^{1}\left(\omega, r_{*}\right)$ and $\bar{Z}_{\ell}^{2}\left(\omega, r_{*}\right)$ will be independent of $m$ as well. We then construct the solution of the Nakamura-Sasaki equation as follows

$$
\begin{aligned}
Z_{\ell m}^{N S}\left(\omega, r_{*}\right) & =\frac{\alpha_{\ell m}(\omega)}{W_{\ell}^{1}}\left[\bar{Z}_{\ell}^{1}\left(\omega, r_{*}\right) \int_{r_{*}}^{\infty} Y_{\ell}\left(\omega, y_{*}\right) S_{\ell m}^{N S}\left(\omega, y_{*}\right) d y_{*}\right. \\
& \left.+Y_{\ell}\left(\omega, r_{*}\right) \int_{R}^{\infty} \bar{Z}_{\ell}^{1}\left(\omega, y_{*}\right) S_{\ell m}^{N S}\left(\omega, y_{*}\right) d y_{*}\right] \\
& +\frac{\left(1-\alpha_{\ell m}(\omega)\right)}{W_{\ell}^{2}}\left[\bar{Z}_{\ell}^{2}\left(\omega, r_{*}\right) \int_{r_{*}}^{\infty} Y_{\ell}\left(\omega, y_{*}\right) S_{\ell m}^{N S}\left(\omega, y_{*}\right) d y_{*}\right. \\
& \left.+Y_{\ell}\left(\omega, r_{*}\right) \int_{R}^{\infty} \bar{Z}_{\ell}^{2}\left(\omega, y_{*}\right) S_{\ell m}^{N S}\left(\omega, y_{*}\right) d y_{*}\right],
\end{aligned}
$$


where $W_{\ell}^{1}(\omega)$ and $W_{\ell}^{2}(\omega)$ are the wronskians

$$
\begin{aligned}
& W_{\ell}^{1}(\omega)=Y_{\ell}^{\prime}\left(\omega, r_{*}\right) \bar{Z}_{\ell}^{1}\left(\omega, r_{*}\right)-Y_{\ell}\left(\omega, r_{*}\right) \bar{Z}_{\ell}^{1^{\prime}}\left(\omega, r_{*}\right), \\
& W_{\ell}^{2}(\omega)=Y_{\ell}^{\prime}\left(\omega, r_{*}\right) \bar{Z}_{\ell}^{2}\left(\omega, r_{*}\right)-Y_{\ell}\left(\omega, r_{*}\right) \bar{Z}_{\ell}^{2^{\prime}}\left(\omega, r_{*}\right),
\end{aligned}
$$

and $\quad \alpha_{\ell m}(\omega)$ are constants. It should be mentioned that, since the source $S_{\ell m}^{N S}\left(\omega, r_{*}\right)$ given in eqs. (3.14)-(3.17) diverges as $\gamma^{-1}$ at the turning point, the actual integration of eq. (4.11) can be performed by switching to the proper time $\tau(r)$.

It is easy to check that the solution (4.11) satisfies the pure outgoing wave condition at infinity, and that the matching condition at the boundary are fulfilled provided the constants $\chi_{\ell m}^{1}(\omega)$ and $\quad \chi_{\ell m}^{2}(\omega)$ are defined as

$$
\begin{aligned}
& \chi_{\ell m}^{1}(\omega)=\frac{\alpha_{\ell m}(\omega)}{W_{\ell}^{1}(\omega)} \int_{R}^{\infty} Y_{\ell}\left(y_{*}, \omega\right) S_{\ell m}^{N S}\left(y_{*}, \omega\right) d y_{*} \\
& \chi_{\ell m}^{2}(\omega)=\frac{\left[1-\alpha_{\ell m}(\omega)\right]}{W_{\ell}^{2}(\omega)} \int_{R}^{\infty} Y_{\ell}\left(y_{*}, \omega\right) S_{\ell m}^{N S}\left(y_{*}, \omega\right) d y_{*} .
\end{aligned}
$$

The solution at radial infinity, to which we are primarily interested, therefore is

$$
\begin{aligned}
& Z_{\ell m}^{N S}[\infty, \omega]=e^{i \omega r_{*}}\left[\frac{\alpha_{\ell m}(\omega)}{W_{\ell}^{1}(\omega)} \int_{R}^{\infty} \bar{Z}_{\ell}^{1}\left(y_{*}, \omega\right) S_{\ell m}^{N S}\left(y_{*}, \omega\right) d y_{*}+\right] \\
& {\left[\frac{\left[1-\alpha_{\ell m}(\omega)\right]}{W_{\ell}^{2}(\omega)} \int_{R}^{\infty} \bar{Z}_{\ell}^{2}\left(y_{*}, \omega\right) S_{\ell m}^{N S}\left(y_{*}, \omega\right) d y_{*}\right] }
\end{aligned}
$$

The constants $\alpha_{\ell m}(\omega)$ will be determined by the following arguments. Due to the properties of the spherical harmonics

$Y_{\ell m}(\vartheta, \varphi)=(-1)^{m} Y_{\ell m}(\vartheta, \varphi+\pi)=(-1)^{\ell} Y_{\ell m}(\pi-\vartheta, \varphi+\pi)=(-1)^{\ell+m} Y_{\ell m}(\pi-\vartheta, \varphi)$,

$Y_{\ell m}(\vartheta, \varphi)=(-1)^{m} Y_{\ell-m}^{*}(\vartheta, \varphi)$

by an inspection of the behaviour of the source $S_{\ell m}^{N S}$, it is easy to verify that the NakamuraSasaki function satisfies the following identity

$$
Z_{\ell-m}^{N S *}(-\omega, r)=(-1)^{\ell} Z_{\ell m}^{N S}(\omega, r)
$$

and consequently 


$$
\begin{aligned}
& Z_{\ell-m}^{1 *}(-\omega, R)=(-1)^{\ell} Z_{\ell m}^{1}(\omega, R) \\
& Z_{\ell-m}^{2 *}(-\omega, R)=-(-1)^{\ell} Z_{\ell m}^{2}(\omega, R) .
\end{aligned}
$$

In addition, by explicitely evaluating $\delta \Psi_{4}(t, r, \vartheta, \varphi)$ for the perturbed metric (2.1), and by using eq. (3.6), we find

$$
\begin{aligned}
D_{C H} Z_{\ell m}^{1} & =\frac{r \sqrt{n(n+1)}}{4}\left\{r e^{2 \nu}\left[N_{\ell m}(\omega, r)-L_{\ell m}(\omega, r)\right]\right. \\
& +r^{3} e^{2\left(\nu-\mu_{2}\right)}\left[-V_{\ell m}^{\prime \prime}(\omega, r)+\left(\mu_{2}^{\prime}+\nu^{\prime}-\frac{2}{r}\right) V_{\ell m}^{\prime}(\omega, r)\right] \\
& \left.-2 i \omega r^{3} e^{\left(\nu-\mu_{2}\right)} V_{\ell m}^{\prime}(\omega, r)+r^{3}\left[\omega^{2}+2 i \omega e^{\left(\nu-\mu_{2}\right)}\left(\nu^{\prime}-\frac{1}{r}\right)\right] V_{\ell m}(\omega, r)\right\} \\
D_{C H} Z_{\ell m}^{2} & =\frac{r \sqrt{n(n+1)}}{4}\left\{\frac { r } { 2 } \left[e^{2\left(\nu-\mu_{2}\right)} h_{\ell m}^{1^{\prime}}(\omega, r)-e^{2\left(\nu-\mu_{2}\right)}\left(\mu_{2}^{\prime}+\nu^{\prime}\right) h_{\ell m}^{1}(\omega, r)+i \omega e^{\left(\nu-\mu_{2}\right)} h_{\ell m}^{1}(\omega, r)\right.\right. \\
& \left.\left.-e^{\left(\nu-\mu_{2}\right)} h_{\ell m}^{0^{\prime}}(\omega, r)+2 \nu^{\prime} e^{\left(\nu-\mu_{2}\right)} h_{\ell m}^{0}(\omega, r)-i \omega h_{\ell m}^{0}(\omega, r)\right]\right\}
\end{aligned}
$$

From the property of the metric perturbations given in eq. (2.5), and since $D_{C H}^{*}(\omega)=$ $D_{C H}(-\omega)$ it follows that

$$
\begin{aligned}
& Z_{\ell m}^{1 *}(\omega, r)=(-1)^{m} Z_{\ell-m}^{1}(-\omega, r) \\
& Z_{\ell m}^{2 *}(\omega, r)=(-1)^{m} Z_{\ell-m}^{2}(-\omega, r) .
\end{aligned}
$$

Eqs. (4.18) and (4.20) are compatible only if

$$
\begin{aligned}
& (-1)^{\ell} Z_{\ell m}^{1}(\omega, R)=(-1)^{m} Z_{\ell m}^{1}(\omega, R) \\
& -(-1)^{\ell} Z_{\ell m}^{2}(\omega, R)=(-1)^{m} Z_{\ell m}^{2}(\omega, R),
\end{aligned}
$$

which imply that

- if $(\ell+m)$ is even, $\quad Z_{\ell m}^{2}(\omega, R)=0$,

- if $(\ell+m)$ is odd, $Z_{\ell m}^{1}(\omega, R)=0$,

From eqs. (4.5) we see that if $(\ell+m)$ is even, in order $Z_{\ell m}^{2}(\omega, R)$ to be zero $\chi_{\ell m}^{2}(\omega)$ must vanish, which means that $\alpha_{\ell m}(\omega)=1$ (see eq. 4.14). Similarly, if $(\ell+m)$ is odd $\chi_{\ell m}^{1}(\omega)$ must vanish, i.e. $\quad \alpha_{\ell m}(\omega)=0$. Thus, the complete solution of the Nakamura-Sasaki equation at radial infinity is (see eq. 4.15) 
- $(\ell+m)$ even

$$
Z_{\ell m}^{N S}[\omega, \infty]=\frac{e^{i \omega r_{*}}}{W_{\ell}^{1}(\omega)} \int_{R}^{\infty} \bar{Z}_{\ell}^{1}\left(\omega, y_{*}\right) S_{\ell m}^{N S}\left(\omega, y_{*}\right) d y_{*}
$$

- $(\ell+m)$ odd

$$
Z_{\ell m}^{N S}[\omega, \infty]=\frac{e^{i \omega r_{*}}}{W_{\ell}^{2}(\omega)} \int_{R}^{\infty} \bar{Z}_{\ell}^{2}\left(\omega, y_{*}\right) S_{\ell m}^{N S}\left(\omega, y_{*}\right) d y_{*}
$$

Equations (4.22) and (4.23) show that the polar and the axial perturbations contribute to the solution $Z_{\ell m}^{N S}$ depending on $(\ell+m)$ being even or odd, respectively. This fact is not surprising. For example, if we consider the non-homogeneous Regge-Wheeler equation written for the process under consideration

$$
\mathbf{L}_{\mathbf{R W}} Z_{\ell m}^{R W}(\omega, r)=S_{\ell m}^{R W}(\omega, r)
$$

where the source explicitely is

$$
\begin{aligned}
S_{\ell m}^{R W}= & \frac{4 \imath m_{0} e^{\nu-\mu_{2}}}{\sqrt{2}} e^{\imath \omega[t(r)]}\left\{\left.\imath e^{2 \nu} \frac{\sqrt{2} n(\ell)}{r^{2}(r-2 M)} L_{z} Y_{\ell m, \vartheta}^{*}\right|_{\vartheta=\pi / 2}+\right. \\
& -\left.2 e^{2 \nu} m \frac{\sqrt{2} m(\ell)}{r^{4}} g(r)^{-1 / 2} L_{z}^{2} Y_{\ell m, \vartheta}^{*}\right|_{\vartheta=\pi / 2}+ \\
& -\left.\frac{m(\ell)}{r} m \tilde{L}^{2} Y_{\ell m, \vartheta}^{*}\right|_{\vartheta=\pi / 2}\left[\frac{d}{d r}\left(r^{2} e^{2 \nu} \frac{\sqrt{2}}{r^{4}} g(r)^{-1 / 2}\right)+\right. \\
& \left.\left.+r^{2} e^{2 \nu} \frac{\sqrt{2}}{r^{4}} g(r)^{-1 / 2}\left(\imath \omega \frac{d t(r)}{d r}-\imath m \frac{d \varphi(t(r))}{d r}\right)\right]\right\},
\end{aligned}
$$

and $g(r)=\left(\frac{2 M}{r}-\frac{r-2 M}{r^{3}} L_{z}^{2}\right), \quad n(\ell)=[\ell(\ell+1)]^{-1}$, and $m(\ell)=[\ell(\ell+1)(\ell+2)(\ell-1)]^{-1}$, when $(\ell+m)$ is even $\left.Y_{\ell m, \vartheta}^{*}\right|_{\vartheta=\pi / 2}=0$, the source term is zero, and the axial perturbations are not excited.

It should be mentioned that the procedure used in this paper to find the constants $\alpha_{\ell m}(\omega)$ exploits the symmetry properties of the source of the Nakamura-Sasaki equation, and is much simpler than that described in Paper II. 


\section{THE MODEL OF STAR}

We have integrated eqs. (2.6) and (2.10) in the interior of a star with a polytropic equation of state $p=K \rho^{n}$, with $K=100 \mathrm{~km}$ and $n=2$. If the central density is chosen to be $\rho_{c}=3 \cdot 10^{15} \mathrm{~g} / \mathrm{cm}^{3}$, the radius and mass of the star are, respectively, $R=8.86 \mathrm{~km}$, and $M=1.266 M_{\odot}$, with a ratio $R / M=4.7$ For this model of star, in Fig. 1 we plot the resonance curve evaluated for the $\ell=2$, polar perturbations, which allows to locate the frequencies of the quasi-normal modes of the star. This curve is obtained by integrating the polar equations inside and outside the star (eqs. 2.6, 2.9) for assigned real values of the frequency, and by evaluating the amplitude of the standing wave, $\left[\alpha^{2}(\omega)+\beta^{2}(\omega)\right]$, prevailing at radial infinity. It can be shown that the values of frequency at which this curve exhibits a sharp minimum correspond to the real part of the complex eigenfrequency of a quasi-normal mode, provided the corresponding imaginary part is small enough $\left(\omega_{i}<<\omega_{0}\right)$ [2], [10]. The damping time associated to a mode is related to the curvature of the parabola that fits the curve near a minimum: smoother minima correspond to shorter damping times.

As in newtonian theory, the classification of the polar modes is based on the behaviour of the perturbed fluid according to the restoring force that is prevailing [11]: the g-modes, or gravity modes, when the force is due to the eulerian change in the density, the p-modes, when it is due to a change in pressure, and the f-mode, that is the generalization of the only possible mode of oscillation of an incompressible homogeneous sphere [12]. An inspection of the behaviour of the thermodynamical variables in correspondence of the minima of the resonance curve shown in Fig. 1, allows to identify the corresponding modes. The resonance curves for $\ell=3$ and $\ell=4$ are plotted in Fig. 2. A resonance curve can be computed also for the axial perturbations by solving eq. (2.10). For the model of star we consider, it exhibits a monotonically decreasing behaviour, showing that no slowly damped axial modes

exist in this case. The algorithm based on the resonance curve allows to find only the slowly damped modes $\left(\omega_{i}<<\omega_{0}\right.$.). To find the highly-damped modes (the w-modes [13]) other 
methods have to be used. In table 1, we tabulate the values of the complex frequency of the first few polar w-modes computed in ref. [13] for the same model of star considered in the present paper. We do not go into more details about these modes because, as we shall see, they are not excited in scattering processes.

\section{NUMERICAL RESULTS}

As discussed in section IV, we find $\bar{Z}_{\ell}^{\text {pol }}(\omega, R)$ and $\bar{Z}_{\ell}^{a x}(\omega, R)$ and their first derivatives by solving the interior equations (2.6) and (2.10) for assigned values of the frequency. With these values we construct $\bar{Z}_{\ell}^{1}(\omega, R)$ and $\bar{Z}_{\ell}^{2}(\omega, R)$, and integrate the homogeneous ReggeWheeler equation for these functions and for the function $Y_{\ell}^{1}\left(\omega, r_{*}\right)$ defined in eq. (4.10), up to radial infinity. These functions are needed to construct the solution of the NakamuraSasaki equation, and find its asymptotic behaviour given by eqs. (4.22) and (4.23). The perturbations are assumed to be excited by a massive particle $m_{0}$, which is scattered by the gravitational field of the star; we set the angular momentum and the energy respectively to $L_{z}=5$, and $E=1.007$. For these values, the turning point is located at $r_{t} / M=9.2$ (case a). We then repeat the calculations allowing the particle to get closer to the star: we choose $E=1.097$, so that $r_{t} / M=5.0$ (case b).

For a plane wave (see for example [14] pg. 522 eq. (488))

$$
\delta \Psi_{4}(t, r, \vartheta, \varphi)=-\frac{1}{2}\left[\ddot{h}_{\varphi \varphi}(t, r, \phi, \theta)+i \ddot{h}_{\vartheta \varphi}(t, r, \phi, \theta)\right]
$$

and since the $(0 r)$-component of the stress-energy pseudotensor can be written as

$$
t_{0 r}=\left(\frac{d E}{d S d t}\right)=\frac{1}{16 \pi}\left\{\left[\dot{h}_{\varphi \varphi}(t, r, \varphi, \vartheta)\right]^{2}+\left[\dot{h}_{\vartheta \varphi}(t, r, \varphi, \vartheta)\right]^{2}\right\}
$$

it follows that the energy emitted in gravitational waves per unit solid angle and unit frequency is

$$
\frac{d E}{d \Omega d \omega}=\frac{r^{2}}{2 \omega^{2}}\left|\delta \Psi_{4}(\omega, r, \vartheta, \varphi)\right|^{2}
$$


By integrating over the solid angle, and by using the relation existing between $\delta \Psi_{4}$ and the Nakamura-Sasaki function and discussed in section III, it can be shown that the energy emitted per unit frequency can be expressed as a function of the amplitude of $Z_{\ell m}^{N S}$ at infinity, i.e.

$$
\frac{d E_{\ell m}}{d \omega}=16 \omega^{2}\left|A_{\ell m}^{N S}(\omega)\right|^{2}, \quad 0<\omega<\infty
$$

where

$$
Z_{\ell m}^{N S}(\omega, \infty) \rightarrow A_{\ell m}^{N S}(\omega) e^{i \omega r_{*}}
$$

and $A_{\ell m}^{N S}(\omega)$ is determined by numerical integration. We find that, as in the case of the scattering of masses by a black hole, the energy spectrum of the emitted gravitational radiation is contributed mainly by the $\ell=m$ component.

In Fig. 3 we plot the $\ell=m=2$ energy spectrum emitted in case a, when the turning point is $r_{t}=9.2 \mathrm{M}$. It is interesting to compare this spectrum with that obtained when a black hole scatters a small mass (see [15] for an extensive review). In that case the energy is emitted essentially by the scattered mass, and most of it is radiated when the mass transits through the turning point. Indeed, the energy spectrum is peaked at a frequency which is related to the angular velocity of the mass at the turning point

$$
\omega_{r_{t}}=\ell\left(\frac{d \varphi}{d t}\right)_{r=r_{t}}
$$

Thus, the black hole quasi-normal modes are not excited in these processes. In our case a, and for $\quad \ell=2$, the frequency corresponding to the angular velocity of the mass at the periastron is $\omega_{r t} M=0.092$ and the spectrum shown in Fig. 3 exhibits a sort of peak close to that frequency, showing that part of the energy is still emitted by the mass as a synchrotron radiation. However the very distinctive feature of that spectrum is a very sharp peak which occurs at the frequency of the fundamental f-mode (cfr. Fig. 1). If we allow the mass to get closer to the star, as shown in Fig. 4 for case b when the turning point is $r_{t}=5$, the amplitude of the f-mode peak increases by more than a factor $10^{2}$, and new 
peaks appear, corresponding to the excitation of the p-modes. (In this case $\omega_{r_{t}} M=0.221$ ). Thus, we can conclude that, unlike the case of black holes, the polar quasi-normal modes of the star can be excited in scattering processes, to an extent that depends on how close the mass can get to the star. Conversely, from table 1 and Fig. 3 and 4, we see that the w-modes are not significantly excited in these processes. It should be mentioned that, although we plot the energy spectra for $\omega M \leq 1$, we have extended our numerical integration at higher frequencies finding that the signal is negligible compared with that shown in the figures and no further modes appear. In Fig. 5 we plot the energy spectrum for $\quad \ell=2$ and different values of $-\ell \leq m \leq \ell$. We see that when $\ell+m$ is odd, no modes are excited, because the spectrum is contributed only by the axial perturbations which are not resonant for this model of star. In Fig. 6 we compare the energy spectrum emitted for $\ell=2,3$ and 4 . The excitation of the quasi-normal modes is exhibited by all multipoles.

\section{CONCLUDING REMARKS}

In 1983 Lindblom and Detweiler investigated the relation existing between the frequencies of oscillation of stars and the equation of state (EOS) prevailing in the interior, showing that quasi-normal modes carry information on the internal structure of a star [16]. They computed the frequency of the fundamental mode of oscillation (the f-mode), for several EOS's, and more recently this study has been extended to include a few modern EOS's suggested for neutron stars, for which also the frequencies of the first p-mode and of the polar and axial w-modes have been computed. Besides, it has been shown that the knowledge of these frequencies allows to infer empirical relations between the mode frequency and the macroscopic parameters of the star: the mass and the radius [17], [18]. Thus, there is no doubt that the observation of the quasi-normal modes would provide relevant information on both the structure of neutron stars and on the nature of the nuclear interactions at supernuclear densities.

The real problem is to ascertain whether these modes can be excited in realistic astro- 
physical situations, and what is the amount of gravitational energy that is emitted at the corresponding frequencies. The work presented in this paper is a first step in this direction. The energy spectra plotted in Fig. 3-6 clearly show that the quasi-normal modes of stars can be excited in scattering processes. The waveforms of the corresponding signals are essentially exponentially damped, pure sinusoids, at the frequency of the f-mode of the considered star $(\nu \sim 3 k H z, \tau \sim 0.07 s)$, and with an amplitude which scales as $m_{0}^{-2}$, and depends on how close the scattered mass gets to the star.

The p-modes can be excited if the mass gets sufficiently close (Fig. 4), but the f-mode contribution is likely to be the dominant one. Thus, the signal emitted by a star excited by a scattered mass is a pure note, emitted at the frequency, and with the damping time, of the fundamental f-mode. The spectra we compute do not show the excitation of the w-modes. However, they may be excited in other processess, like for example the capture of masses by stars.

We plan to extend the present work, to compute the energy and the waveforms emitted by masses orbiting around compact stars.

\section{ACKNOWLEDGMENTS}

We gratefully acknowledge Dr. Emanuele Leonardi and the whole INFN CONDOR group for introducing us to CONDOR, a procedure which allowed us to access INFN computational facilities distributed throughout Italy. 


\section{REFERENCES}

[1] M.Davis, R.Ruffini, W.H.Press, R.H.Price, Phys. Rev. Lett. 27, 1466 (1971)

[2] S.Chandrasekhar, V.Ferrari, Proc. R. Soc. Lond. A432, 247 (1990)

[3] T.Regge, J.A.Wheeler, Phys. Rev. 108, 1063 (1957).

[4] J.F. Zerilli, Phys. Rev D. 2, 2141 (1970)

[5] T.Nakamura, M.Sasaki Phys. Lett. 87A, 85 (1981)

[6] V. Ferrari, L.Gualtieri, Int. J. Mod. Phys. D 6 n. 3, 323 (1997)

[7] J.M.Bardeen, W.H.Press, J. Math. Phys. 14, 7 (1973)

[8] S.A.Teukolsky, Ap. J. 185, 635 (1973)

[9] S.Chandrasekhar, Proc. R. Soc. Lond. A343, 289 (1975)

[10] S.Chandrasekhar, V. Ferrari, R. Winston, Proc. R. Soc. Lond. A434, 635 (1991)

[11] T.G. Cowling, MNRAS 101, 367 (1942)

[12] S.Chandrasekhar, Ap. J. 140 n. 2, 417 (1964)

[13] K.D. Kokkotas, B.F. Schutz, 1992, MNRAS 255, 119 (1992)

[14] S. Chandrasekhar The mathematical theory of black holes", Oxford: Claredon Press (1984)

[15] T.Nakamura, K. Oohara, Y. Kojima, Prog. Theor. Phys. Suppl. 90, 1 (1987)

[16] L. Lindblom, S.L. Detweiler, Ap. J. Suppl. 53, 73 (1983)

[17] N. Andersson, K.D. Kokkotas, MNRAS 299, 1059 (1998)

[18] E. Berti, O. Benhar, V. Ferrari, submitted to MNRAS (1999), preprint gr-qc/9901037 


\section{FIGURES}

FIG. 1. The $\ell=2$ - polar resonance curve is plotted versus the normalized frequency $\omega M$. Each minimum corresponds to a quasi-normal mode, and the modes are labelled according to the Cowling classification.

FIG. 2. The $\ell=3$ (right) and $\ell=4$ (left) polar resonance curves are plotted as a function of the frequency as in fig. 1.

FIG. 3. The $\ell=m=2$ - component of the energy spectrum of the gravitational radiation emitted when a mass $m_{0}$, with $L_{z}=5$ and $E=1.007$, is scattered by a polytropic star, is plotted versus the normalized frequency. In this case the turning point is located at $r_{t} / M=9.2$ (case a).

FIG. 4. The $\ell=m=2$ - component of the energy spectrum emitted when the scattered mass $m_{0}$ has an energy at infinity, $E=1.097$, higher than that considered in fig. 1 . In this case the turning point is located at $r_{t} / M=5.0$, and the mass gets closer to the star (case b).

FIG. 5. The $m$-components of the energy spectrum are plotted for $\quad \ell=2$ for case $\mathrm{b}$. We see that when $(\ell+m)$ is even, the polar quasi-normal modes are excited. When $(\ell+m)$ is odd, the energy is emitted essentially by the scattered mass (see text).

FIG. 6. The energy spectrum emitted at $\ell=2$ is compared with that emitted by higher multipoles (case b). 


\section{TABLES}

TABLE I. The values of the real and imaginary part of the frequencies of the first few polar w-modes for the model of star under consideration.

\begin{tabular}{lcr}
\hline \hline$N$ & $\operatorname{Re}(\omega M)$ & $\operatorname{Im}(\omega M)$ \\
\hline 1 & 0.53 & 0.30 \\
2 & 0.88 & 0.38 \\
3 & 1.22 & 0.43 \\
4 & 1.57 & 0.47 \\
5 & 1.91 & 0.50 \\
\hline \hline
\end{tabular}




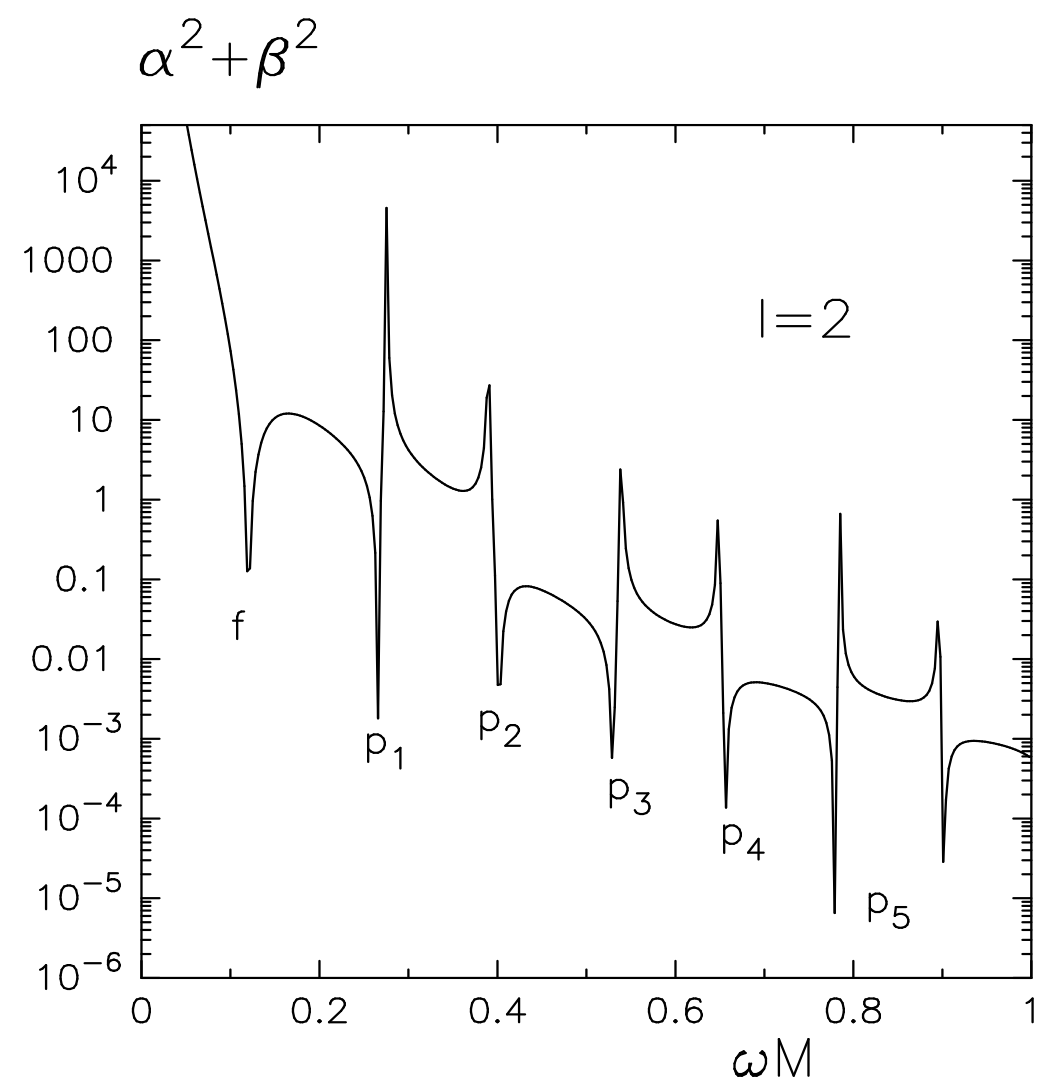



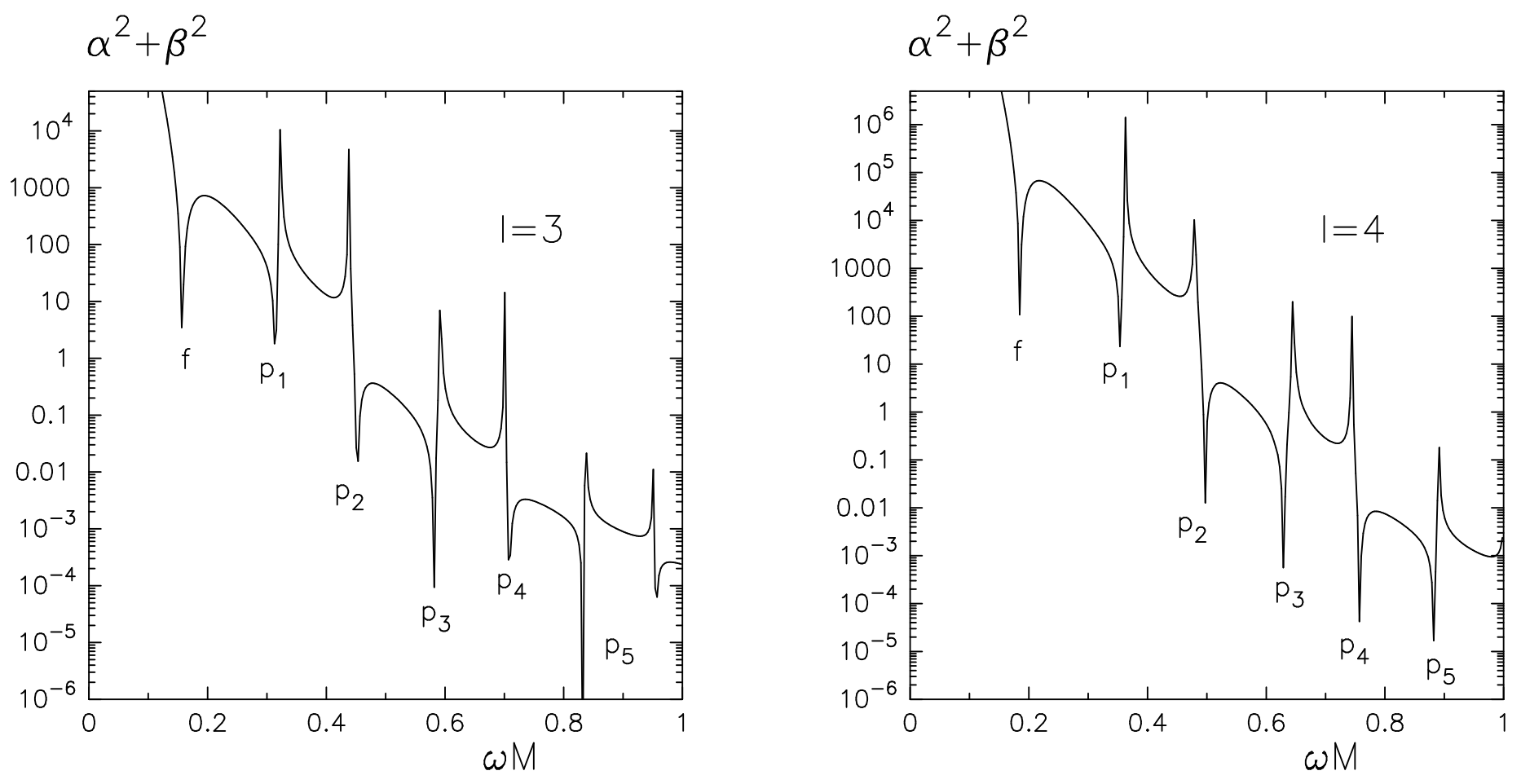


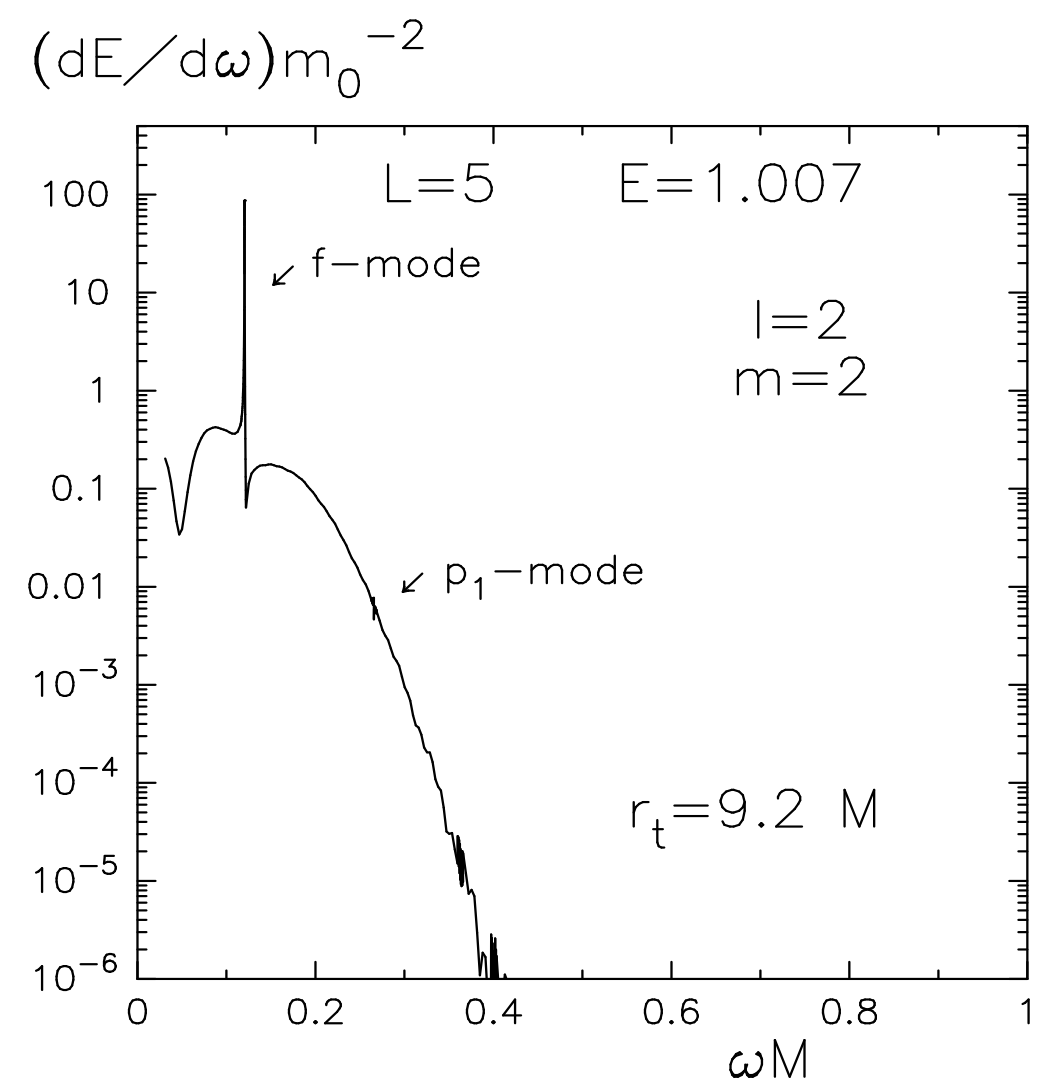




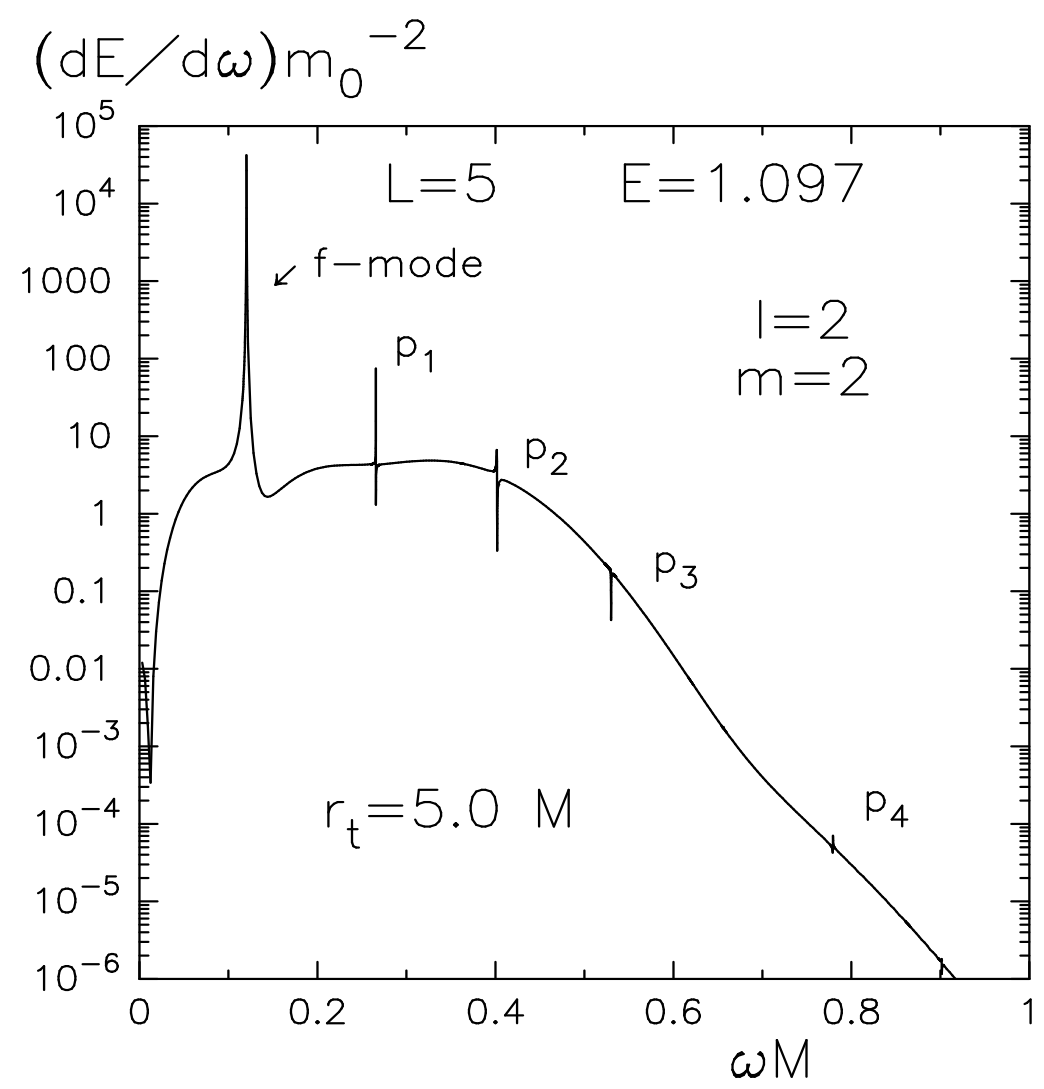



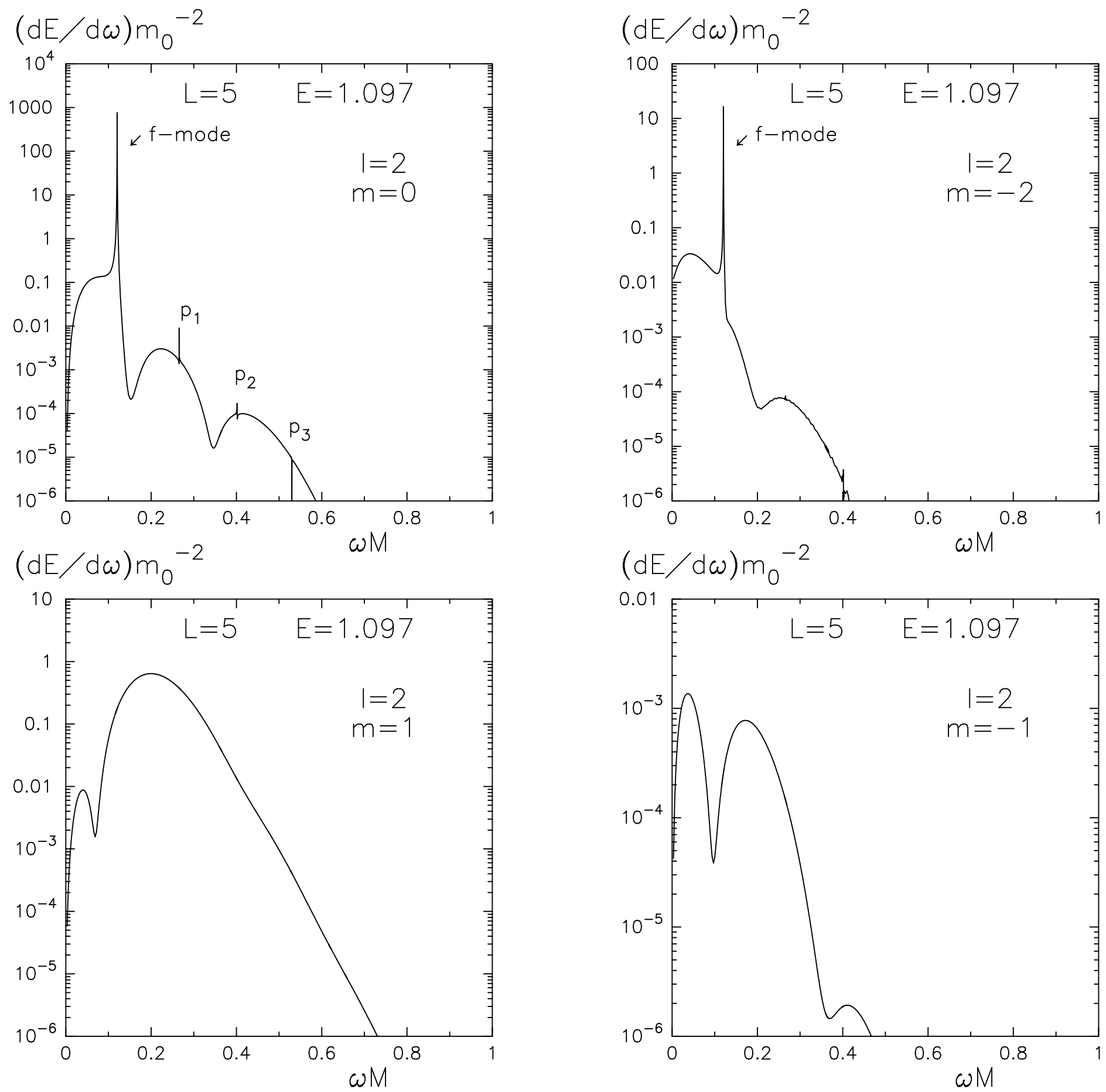


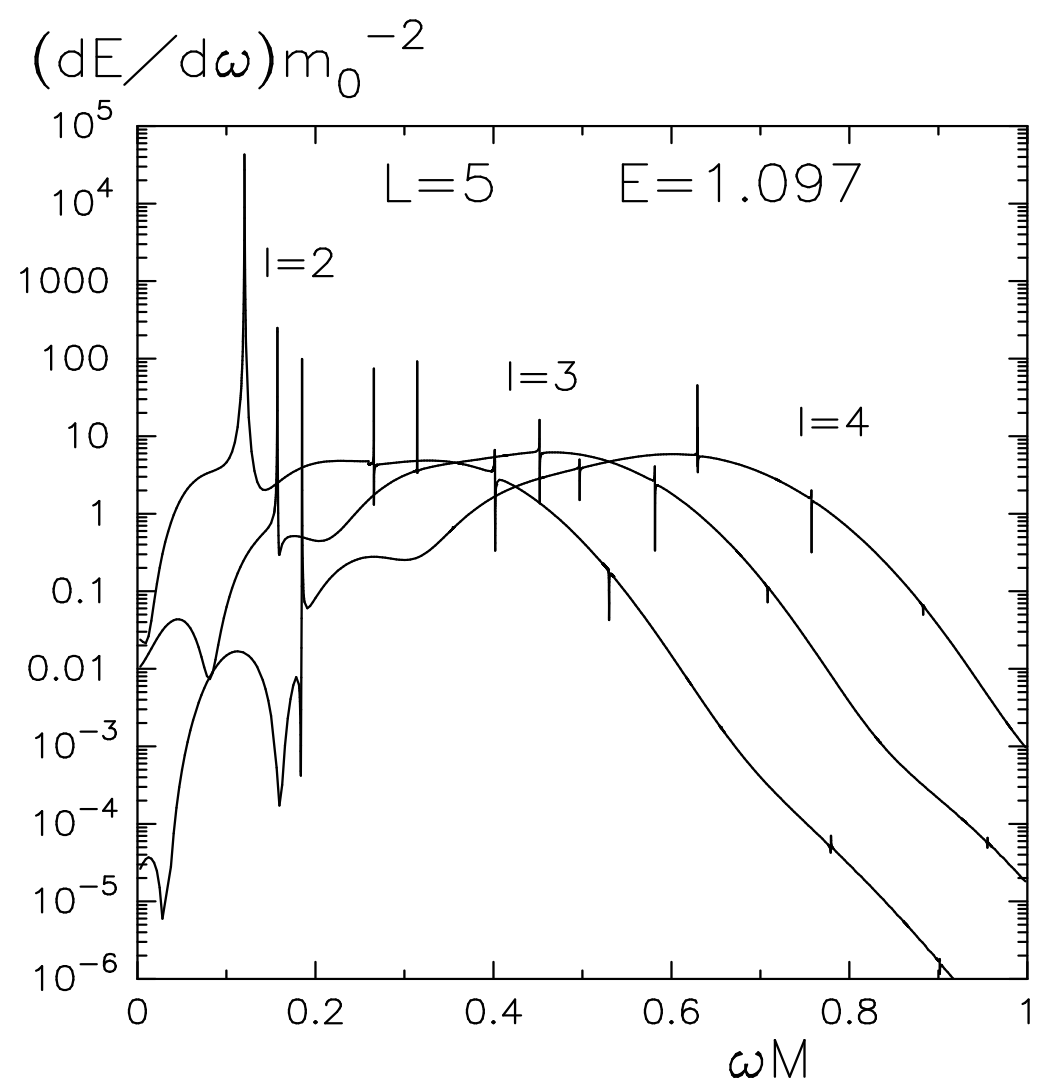

